\title{
DNA knotting in spooling like conformations in bacteriophages
}

\author{
J. Arsuaga ${ }^{\mathrm{a}, \mathrm{b} 1} *$ and Y. Diao ${ }^{\mathrm{c} 2}$ \\ ${ }^{a}$ Department of Mathematics, San Francisco State University, San Francisco, CA, USA; ${ }^{b}$ Institute for \\ Mathematics and Its Applications, University of Minnesota, Minneapolis, MN, USA; ${ }^{c}$ Department of \\ Mathematics and Statistics, University of North Carolina at Charlotte, Charlotte, NC, USA
}

(Received 13 February 2008; final version received 3 April 2008)

\begin{abstract}
A number of idealized models have been proposed to explain the long range organization of the DNA in bacteriophages. However, none of these models can account for the distributions of complex knots found when examining DNA extracted from bacteriophage P4 capsids. Furthermore, these models do not consider possible chirality biases in the arrangement of the DNA molecule inside the capsid. In this paper, we address these two issues by proposing a randomized version of one of the most popular models: the coaxially spooled model. We present analytical and numerical results for the properties of the random polygons (knots) generated using this model. We show that such model can easily generate complex knotted conformations and although it accounts for some chirality of the organization of the DNA molecules inside bacteriophage capsids does not fully explain the experimental data.
\end{abstract}

Keywords: random polygons; random knots; bacteriophage; DNA packing; spooling model

AMS Subject Classification: 57M25; 92Bxx

\section{Introduction}

All icosahedral bacteriophages with double-stranded DNA genomes pack their chromosomes in a similar manner [13] and are believed to hold similar DNA arrangements as those found in herpes viruses [8] as well as in lipo-DNA complexes used in gene therapy [34]. Most bacteriophages consist of an icosahedral capsid that contains the nucleic acid (i.e. DNA or RNA), a tail with tail fibres, and a connector that allows the assembly of the capsid and the tail [33]. During phage morphogenesis a proteinic procapsid is first assembled (e.g. [43]). This is followed by the transfer of one copy of the viral genome inside the capsid through the connector (e.g. [36]). At the end of the packing process the volume of the DNA molecule is reduced $10^{2}$ times [18] reaching concentration levels of $800 \mathrm{mg} / \mathrm{ml}$ [19] and an osmotic pressure of $70 \mathrm{~atm}$ $[14,40]$. A number of models have been proposed to explain the global organization of DNA inside phage capsids. These include the ball of string model [32], the coaxial and concentric spooling models [2,9,13,32,35], the spiral-fold model [7], the folded toroidal model [17], the twisted toroidal model [30] and liquid crystalline models (e.g. [21]). Spooling and toroidal models are the most accepted in the biological community $[2,9,13,32,33,35]$ however, liquid crystalline models are somewhat more consistent with local properties of the DNA molecule under extreme conditions of pressure [37] and have been proposed for chromosome organization in other viruses [8]. Here, we investigate if spooling like conformations are consistent with data obtained from bacteriophage P4 capsids.

\footnotetext{
*Corresponding author. Email: jarsuaga@ @fsu.edu
} 
Bacteriophage P4 has an icosahedral capsid $(T=4,[12])$ and its genome is a doublestranded DNA genome, $10-11.5 \mathrm{~Kb}$ in length, flanked by $16 \mathrm{bp}$ single-stranded ends that are complementary in sequence [42]. The packaged genome is preserved as a linear molecule [31] by keeping at least one of its ends attached to the connector region [10]. However, a high percentage of DNA molecules artificially extracted from bacteriophage P4 are closed circular knots. Furthermore, most of these knots have an elevated knot complexity. This phenomenon is further magnified in P4 tailless mutants [3,22,23] and in naturally occurring deletion mutants [44]. In Ref. [3] it was shown that the majority of the knots are formed inside the viral capsid and therefore may be used as reporters for the DNA organization inside the capsid. In fact, analysis of knots of up to seven crossings revealed a very distinct distribution of knots. Some of the characteristics include the absence of the four crossing knot (called the figure eight knot in knot theory) and a prevalence of toroidal knots (i.e. knots that can be constructed on the surface of a torus) over twist knots $[4,39]$. These findings suggested a chiral 3D organization of the viral genome [4].

Current idealized models have rarely been confronted with these data and, if so they have been unable to reproduce such knot distributions. For instance in Refs. [2,20] molecular dynamics simulations of the coaxially and concentrically spooling models were unsuccessful in obtaining knotted conformations. On the other hand, models that explain knotting under these conditions of confinement $[3-5,26,27]$ show very unrealistic conformations for the viral genome and are far from those models proposed by other groups. This discrepancy between DNA packing models and knotting of DNA in P4 phages motivates this study.

In this paper, we present a randomized spooling model and investigate the knotting and chirality of the generated configurations. First, we introduce the essential mathematical concepts needed for understanding this paper and briefly describe the previously published experimental results. Second, we describe the spooling random polygon. Next, we provide some analytical estimates of the complexity and of the relationship between the spooling of a random polygon with its knotting probability. Finally, we perform numerical studies to compute the knotting probability and complexity.

\section{Methods}

In this section, we will first provide some basic mathematical background on knot theory. We will then give a detailed description of our model. After that, we will talk about what mathematical quantities we would like to estimate and how we will carry out our simulations to compute these quantities.

\subsection{Basic mathematical background}

The most relevant topological concepts used in this paper are given in this subsection. For a more detailed exposition of the basics of knot theory please refer to a standard text such as $[1,24]$.

A knot $K$ is a simple closed curve in $R^{3}$. In this paper, we assume that such a curve is a piecewise smooth curve (this includes a space polygon without self-intersections). For a fixed knot $K$, a regular projection of $K$ is a projection of the simple closed curve onto a plane, such that not more than two segments of $K$ cross at the same point in the projection. If the over/under information of the strands at each intersection is kept, then the projection is called a knot diagram. A fundamental theorem in knot theory states that two knots $K_{1}$ and $K_{2}$ are topologically equivalent, if and only if any regular diagram of $K_{1}$ can be changed to a regular diagram of $K_{2}$ through a sequence of Reidemeister moves, as illustrated in Figure 1. An intersection in a knot diagram is called a crossing. The minimum number of crossings among all possible knot 

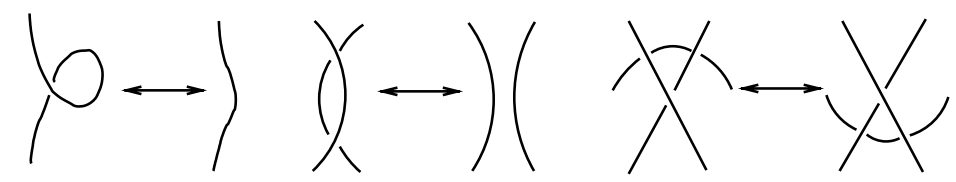

Figure 1. Reidemeister moves.

diagrams that are topologically equivalent to $K$ is called the crossing number of $K$ and is denoted by $\mathrm{Cr}(K)$. The average number of crossings over all possible regular projections of $K$ (as a fixed space curve) is called the average crossing number ( $\mathrm{ACN})$ of $K$ and is denoted by $\mathrm{ACN}(K)$. Note that $\operatorname{Cr}(K)$ is a knot invariant, since it is the minimum number of crossings among all possible knot diagrams that are topologically equivalent to $K$, but not $\operatorname{ACN}(K)$, as it is a quantity that would be affected by a geometric (not topological) change of $K$.

An important concept in this paper is that of chirality. A knot $K$ is said to be achiral, if it is topologically equivalent to its mirror image, otherwise, it is said to be chiral. For example, the right-handed trefoil is topologically distinct from its mirror image (which is the left-handed trefoil). On the other hand, the only four crossing knot $4_{1}$ (also called the figure eight knot) is achiral. If each crossing in a diagram of $K$ is assigned a +1 or -1 , following the sign convention shown in Figure 2, then the summation of these \pm 1 's is called the writhe of this diagram. For example, the writhe of the minimum projection of the knot $4_{1}$ is zero, whereas the writhe of the minimum projection of the right-handed trefoil is +3 (see Figure 2). The average writhe of the knot $K$ (as a space curve), on the other hand, is the average of the writhe over all its regular projection diagrams. In the literature, the average writhe is often just called writhe. However, in this paper, we will make a distinction to avoid the possible confusion. Therefore, the writhe always refers to a single knot projection and the average writhe of $K$ is the average of the writhe over all regular diagrams of $K$. If the writhe were a knot invariant, one would easily conclude that the writhe of an achiral knot projection must be zero since the writhe of a knot diagram changes sign upon reflection. Unfortunately, this is not the case. For example the trivial knot can have a writhe as high as one wants, since a simple super-coil is an unknot and can have as many twists as one wants (which gives a writhe as high as one wants). However, the writhe still provides a measure for the chirality of a knot in the average sense: if two random knots were to be generated by the same generating method, but with fixed knot types, so that one of them is chiral and the other is achiral, then the mean of the average writhe of the achiral random knots would tend to be closer to zero. In this sense, one may argue that the chirality of a (random) knot can be quantified by its writhe. Furthermore, the impact of writhe on the chirality of a knot is more visible for knots with certain structure, as we shall see from Theorem 3.3 and its consequences. An important concept connecting the writhe with the knottedness of $K$ (which is used in Theorem 3.3) is that of Seifert circles. If every crossing in a knot diagram is split as shown at the left side of Figure 3, then the result is a collection of disjoint (topological) circles called the Seifert circles of the knot diagram, see Figure 3.

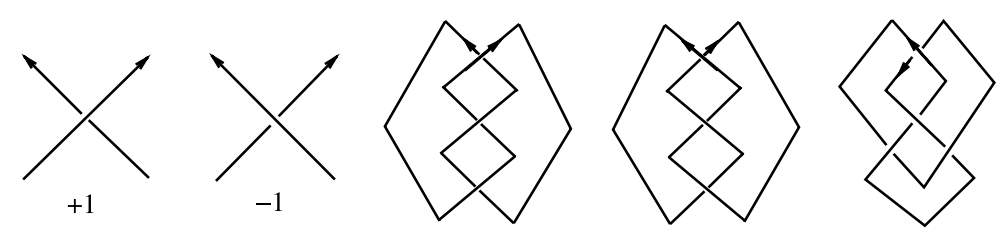

Figure 2. The crossing sign convention and the projections of the right-handed, left-handed trefoils and the figure eight knot whose writhes are $3,-3$ and 0 , respectively. 

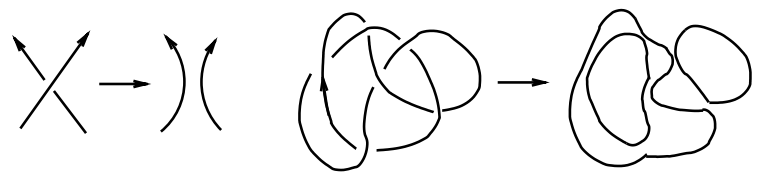

Figure 3. Splitting a knot diagram into Seifert circles.

\subsection{Existing experimental data}

This study is motivated by the data previously published in Refs. [3,4,22,23,38,39,44]. In these experiments, bacteriophages were grown in Escherichia coli cells lysogenic for P2. Tailless mutants were grown on a second strain, also lysogenic for P2, deficient for the genes encoding for the tail and tail fibres. Tailless mutants were purified and DNA was phenol extracted and chloroform purified. DNA samples were run in high resolution 2D gel electrophoresis revealing a large quantities of knots $(\approx 95 \%)$. Quantification of the knot populations of up to seven crossings revealed the absence of four crossings knots, as well as a (yet unidentified) population of six and seven crossing knots. The data also showed a prevalence of toroidal knots for five and seven crossing knots, and the absence of five crossing twist knots.

\subsection{Definition of the coaxially spooled random polygon model}

In this model, the DNA is modelled as an equilateral random polygon and the capsid is modelled by a sphere $S$ of radius $R$ (in bond length units). In this sphere, there is a cylinder of radius $r$ running through the centre. Vertices of the polygon are not allowed to enter the cylinder. The addition of this cylinder in our model is motivated by the predictions of Odijk [29] and by the spaced occupied by the core proteins in the capsid (e.g. [30]); it is also used as an ad hoc parameter to control the rigidity of the polygons when generating spooling conformations. In order to generate spooling conformations, two spherical caps of height

$$
R-\sqrt{R^{2}-(r+1)^{2}}
$$

are also defined. Polygons are not allowed to enter these caps. See Figure 4 for an illustration of the model and of the spherical caps. In order to generate a random polygon satisfying the above conditions these steps are followed. A coordinate system is set so that the original point is the center of $S$ and the $z$-axis is the axis of $T$. For simplicity, we may always choose the starting point whose projection is on the $x$-axis; and without loss of generality, we will use anticlockwise spin
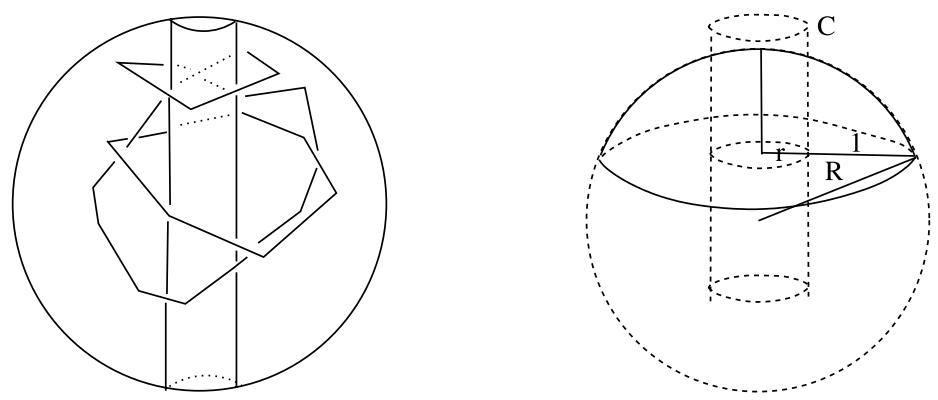

Figure 4. Left: A random spooling DNA packing model. Right: The cap to be avoided by the vertices of the random polygon. 
packing. Name the vertices of the polygon to be $X_{0}, X_{1}, X_{2}, \ldots, X_{n}$. Once $X_{k}$ has been generated, we will follow the following procedure to generate $X_{k+1}$. Let the $z$-coordinate of $X_{k}$ be $z_{k}$ and consider the plane parallel to the $x y$-plane. It intersects $S$ at a circle $C_{k}$ whose radius $r^{\prime}$ is at least $r+1$. Let

$$
r_{k}=\sqrt{x_{k}^{2}+y_{k}^{2}}
$$

where $X_{k}=\left(x_{k}, y_{k}, z_{k}\right)$. Let us try to determine

$$
r_{k+1}=\sqrt{x_{k+1}^{2}+y_{k+1}^{2}},
$$

first. Here, we will introduce the third parameter $h$, which is a small positive number. For the time being, let us assume that it is a number set between 0.1 and 0.5. Basically, a larger $h$ allows more variation in the polygon in its distance to the centre of the cylinder, as well as in the difference of the $z$-coordinates between the end points of each edge. We consider the following three cases.

Case 1. $r+h \leq r_{k} \leq r^{\prime}-h$. In this case, we will choose $r_{k+1}$ from the interval $\left[r_{k}-h\right.$, $\left.r_{k}+h\right]$ uniformly.

Case 2. $r_{k}<r+h$. In this case, we will choose $r_{k+1}$ from the interval $[r, r+2 h]$ uniformly. Case 3. $r_{k}>r^{\prime}-h$. In this case, we will choose $r_{k+1}$ from the interval $\left[r^{\prime}-2 h, r^{\prime}\right]$ uniformly.

Once $r_{k+1}$ is determined, we will go ahead to determine $z_{k+1}$. This is done similarly to the above in three different cases. First set

$$
t_{k}=\min \left\{\sqrt{R^{2}-(r+1)^{2}}, \sqrt{R^{2}-r_{k}^{2}}\right\} .
$$

Case $1^{\prime} .-t_{k}+h \leq z_{k} \leq t_{k}-h$. In this case, we will choose $z_{k+1}$ from the interval $\left[z_{k}-h\right.$, $\left.z_{k}+h\right]$ uniformly.

Case $2^{\prime} . z_{k}>t_{k}-h$. In this case, we will choose $z_{k+1}$ from the interval $\left[t_{k}-2 h, t_{k}\right]$ uniformly.

Case $3^{\prime} \cdot z_{k}<-t_{\mathrm{k}}+h$. In this case, we will choose $r_{k+1}$ from the interval $\left[-t_{k},-t_{k}+2 h\right]$ uniformly.

Now, we just need to find how much rotation we need to take from the ray $\overrightarrow{O_{k} X_{k}}$ (where $\left.O_{k}=\left(0,0, z_{k}\right)\right)$ in the anticlockwise direction (which will then completely determine the coordinates of $\left.X_{k+1}\right)$. Under the condition that $\left|X_{k} X_{k+1}\right|=1$, that angle is given by

$$
\theta_{k}=\cos ^{-1}\left(\frac{r_{k}^{2}+r_{k+1}^{2}+z^{2}-1}{2 r_{k} r_{k+1}}\right)
$$

where $z=z_{k+1}-z_{k}$. See Figure 5 for an illustration of this process.

And, we have

$$
x_{k+1}=r_{k+1} \cos \left(\sum_{1 \leq j \leq k} \theta_{j}\right), \quad y_{k+1}=r_{k+1} \sin \left(\sum_{1 \leq j \leq k} \theta_{j}\right) .
$$




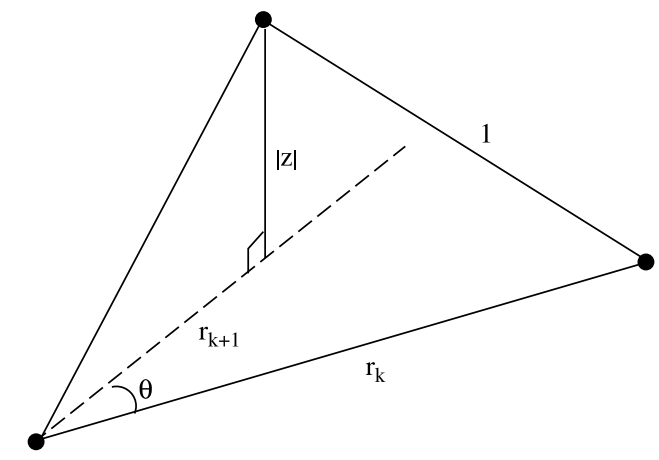

Figure 5. Generating $X_{k+1}$ given $X_{k}$.
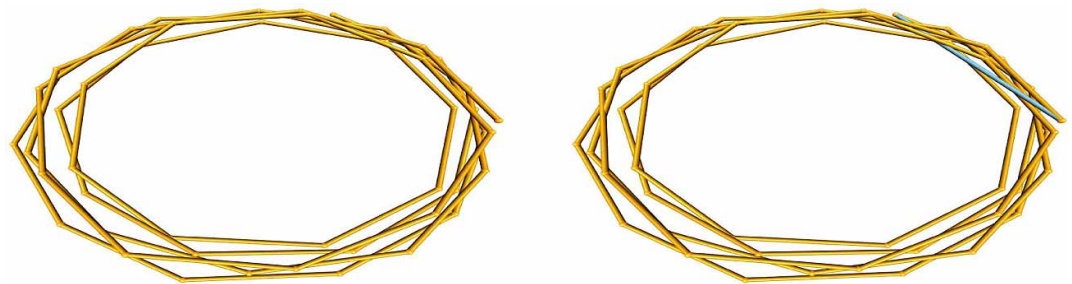

Figure 6. Left: A coaxially spooled equilateral random walk. Right: A forced closure of the random walk at left by a straight-line segment. This figure was generated by R. Scharein using Knot plot. Available in colour online.

(Keep in mind that $X_{1}$ started on the $x$-axis). Figure 6 shows a random polygon generated by this algorithm. A polygon with a few segments is shown to illustrate the natural entanglement of such configurations.

We call the polygons generated in the above process spooling random polygons. A spooling random polygon with $n$ edges is denoted by $P_{n}^{s}$.

\subsection{Determination of the rounding number}

In this model, we can easily control the number of times the polygon will go around the $z$-axis (the rounding number). Say, we want to generate a random polygon that goes around the $z$-axis $m$ times, then we will stop the generating process at the first $n$ such that $\sum_{1 \leq j \leq n} \theta_{j} \geq 2 m \pi$ and replace that last generated point $X_{n+1}$ with $X_{1}$, resulting therefore in a polygon. The last edge of the polygon is no longer of unit length. Although it is conceivable that for a large polygon this probably won't affect its topological property that much, a biologically meaningful way to close the two open ends may still be needed for a more in depth study in the future. In this paper, we will trade off this imperfection for simplicity and speed.

\subsection{Computation of the knotting probability and complexity}

Each polygon is projected along the $z$-axis. The Dowker code of the resulting diagram is computed and it is simplified using Reidemeister I and II moves. From the simplified Dowker code the unknotted trajectories are identified by computing the Alexander polynomial evaluated at $t=-1$ (denoted by $\Delta(-1)$ ). This identification is based on the fact that the trivial knot has $\Delta(-1)=1$. Doing so can cause some error: it is known that $\Delta(-1)$ does not identify all knotted polygons (i.e. $\Delta(-1)=1$ for some non-trivial knots). However, this error is not large since relatively few knots have the property $\Delta(-1)=1$. The advantage of using $\Delta(-1)$ (or more 
generally, the Alexander polynomial $\Delta(t)$ itself) is that the computation of the polynomial is equivalent to the computation of the determinant of a certain matrix, which can be done in polynomial time on the number of crossings. Identification of the knots with low crossing number was done by calculating $\Delta(-2)$.

\subsection{Computation of the average writhe and $A C N$}

The average writhe is a geometric measure of self-entanglement which measures non-planarity of a spatial conformation. The average writhe of a closed curve $C$ can be defined using the Gauss double integral

$$
\frac{1}{4 \pi} \int_{0}^{L} \int_{0}^{L} \frac{(\dot{\gamma}(t), \dot{\gamma}(s), \gamma(t)-\gamma(s))}{|\gamma(t)-\gamma(s)|^{3}} \mathrm{~d} t \mathrm{~d} s,
$$

where $\gamma$ is an equation of $C$ parameterized by arclength, $L$ is the length of $C$ and $(\dot{\gamma}(t), \dot{\gamma}(s), \gamma(t)-\gamma(s))$ is the triple scalar product of $\dot{\gamma}(t), \dot{\gamma}(s)$, and $\gamma(t)-\gamma(s)$. For a given projection, the sign of the crossing is determined using the convention illustrated in Figure 2. At a positive crossing, one can think of the integrand as the measure of the solid angle created by those projections from which the tangent vectors appear to cross. If the crossing is a negative one, the integrand is equal to the measure of this solid angle multiplied by -1 . This interpretation of the integrand allows for an easy calculation of the writhe when $C$ is a polygon of $n$ segments of unit length. In this case, the Gauss integral in (1) can be written as a double sum over the segments of the polygon:

$$
\sum_{1 \leq i \neq j \leq n} \frac{1}{4 \pi} \int_{0}^{1} \int_{0}^{1} \frac{\left(\dot{\gamma}_{i}(t), \dot{\gamma}_{j}(s), \gamma_{i}(t)-\gamma_{j}(s)\right)}{\left|\gamma_{i}(t)-\gamma_{j}(s)\right|^{3}} \mathrm{~d} t \mathrm{~d} s
$$

where $\gamma_{i}$ and $\gamma_{j}$ are expressions for the edges $\ell_{i}$ and $\ell_{j}$ of $C$ parameterized by arclengths. Similarly, the ACN of $C$ is given by Ref. [15]

$$
\frac{1}{4 \pi} \int_{0}^{L} \int_{0}^{L} \frac{|(\dot{\gamma}(t), \dot{\gamma}(s), \gamma(t)-\gamma(s))|}{|\gamma(t)-\gamma(s)|^{3}} \mathrm{~d} t \mathrm{~d} s .
$$

And again, in the case that $C$ is a polygon of $n$ segments of unit length, its ACN can be expressed as

$$
\sum_{1 \leq i \neq j \leq n} \frac{1}{4 \pi} \int_{0}^{1} \int_{0}^{1} \frac{\left|\left(\dot{\gamma}_{i}(t), \dot{\gamma}_{j}(s), \gamma_{i}(t)-\gamma_{j}(s)\right)\right|}{\left|\gamma_{i}(t)-\gamma_{j}(s)\right|^{3}} \mathrm{~d} t \mathrm{~d} s .
$$

\section{Theoretical results}

The following theorem concerns the mean crossing number of a spooling random polygon.

THEOREM 3.1. Let $P_{n}^{s}$ be a spooled random polygon of $n$ edges, then the average number of crossings in its projection to the $x y$-plane perpendicular to its centre axis is of the order of $O\left(n^{2}\right)$.

Proof. Here is an outline of the proof of this lemma. Let $\ell_{1}, \ell_{2}, \ldots, \ell_{n}$ be the consecutive edges of $P_{n}^{s}$ and let $a_{i j}=a\left(\ell_{i}, \ell_{j}\right)$ be the ACN between $\ell_{i}$ and $\ell_{j}$, then the ACN of $P_{n}$ (written as $\chi_{n}$ ) is simply the sum of the $a\left(\ell_{i}, \ell_{j}\right)$ 's: $\chi_{n}=\sum_{1 \leq i<j \leq n} a_{i j}=1 / 2 \sum_{1 \leq i, j \leq n} a_{i j}$, and $E\left(\chi_{n}\right)=$ $\left(\chi_{n}\right)=\sum_{1 \leq i<j \leq n} E\left(a_{i j}\right)=1 / 2 \sum_{1 \leq i, j \leq n} E\left(a_{i j}\right)$, where $a_{i j} / 2$ has the integral form as given 
in (4). For any $j>i+1$ and any fixed $\ell_{i}$, there is a positive probability that the starting point of $\ell_{j}$ is of a small (constant) distance away from $\ell_{i}$ due to the way $\ell_{j}$ is generated. Since $\ell_{i}$ and $\ell_{j}$ are confined in the same sphere of radius $R, \ell_{j}$ cannot be too far away from $\ell_{i}$ either. So, the denominator in the integrand of (4) is bounded above by a constant. Since the end point of $\ell_{j}$ is independent of the position of $\ell_{i}$, there is a positive probability that the numerator of the integrand of (4) is bigger than a constant (however small it may be). It follows that $E\left(a_{i j}\right) \geq b$ for some positive constant $b$ as long as $\ell_{i}$ and $\ell_{j}$ are not adjacent edges. This implies that $E\left(\chi_{n}\right)=1 / 2 \sum_{1 \leq i, j \leq n} E\left(a_{i j}\right) \geq b n(n-3) / 2=O\left(n^{2}\right)$.

Remark. It must be pointed out that the positive constant $b$ mentioned in the above proof can vary greatly depending on the parameters of the model as it is shown in Figure 9. However, the $O\left(n^{2}\right)$ behaviour is clearly seen in the numerical study as shown in Figure 9.

Let us now concentrate on the projection of $P_{n}^{s}$ onto the $x y$-plane. Call this projection $D_{n}$. Essentially, $D_{n}$ is treated as a knot diagram and we can compute $w\left(D_{n}\right)$, the writhe of $D_{n}$. Because of the special structure of $P_{n}^{s}$, we have the following result as well. The proof of this is elementary. An interested reader can convince him/her self by working out a few examples.

THEOREM 3.2. Let $D_{n}$ be the projection of a $P_{n}^{s}$ onto the $x y$-plane and let $\sigma\left(P_{n}^{s}\right)$ be the rounding number of $P_{n}^{s}$ (namely the number of times wraps around its central axis), then the number of Seifert circles in $D_{n}$ is exactly $\sigma\left(P_{n}^{s}\right)$.

The following important theorem is due to Morton. The original theorem was stated for a general diagram using the number of Seifert circles in the place of $\sigma\left(P_{n}^{s}\right)$. For the original version of the theorem and a proof of it, please refer to Ref. [28].

THEOREM 3.3. [28] Let $w\left(D_{n}\right)$ be the writhe of $D_{n}, e$ be the smallest power of $v$ in the HOMFLY polynomial $P_{D_{n}}(v, z)$ of $D_{n}$ (which is the same as the HOMFLY polynomial of $P_{n}^{s}$, of course) and $E$ be the largest power of $v$ in $P_{D_{n}}(v, z)$, then, we have $w\left(D_{n}\right)-\left(\sigma\left(P_{n}^{s}\right)-1\right) \leq e \leq E \leq$ $w\left(D_{n}\right)+\left(\sigma\left(P_{n}^{s}\right)-1\right)$.

An immediate consequence of this theorem is the following important corollary. It reveals a very nice relation between the writhe of $D_{n}$ and the chirality of $P_{n}^{s}$.

CoRollary 3.4. [28] If $\left|w\left(D_{n}\right)\right| \geq \sigma\left(P_{n}^{s}\right)$, then $P_{n}^{s}$ is a non-trivial knot. Furthermore, in this case $P_{n}^{s}$ cannot be an achiral knot.

Proof. We have $e=0=E$ for the unknot (by the definition of the HOMFLY polynomial). Yet if $\left|w\left(D_{n}\right)\right| \geq \sigma\left(P_{n}^{s}\right)$, then either $e \geq 1$ or $E \leq-1$. On the other hand, if $P_{n}^{s}$ is an achiral knot, then it is equivalent to its mirror image. Since $P_{K}(v, z)=P_{K^{\prime}}\left(v^{-1}, z\right)$ when $K^{\prime}$ is the mirror image of $K$, it follows that $P_{D_{n}}(v, z)=P_{D_{n}}\left(v^{-1}, z\right)$. Therefore, $e=-E$ so, we must have $e \leq 0 \leq E$. But again, if $w\left(D_{n}\right) \geq \sigma\left(P_{n}^{s}\right)$, we have $1 \leq e$ and if $w\left(D_{n}\right) \leq-\sigma\left(P_{n}^{s}\right)$, we have $E \leq-1$, leading to contradictions in both cases. Thus, $P_{n}^{s}$ has to be a chiral knot.

This corollary clearly shows that if $P_{n}^{s}$ is knotted because of its high writhe (as initially proposed in Ref. [4]), then it simply cannot be an achiral knot. So, if the DNA in a bacteriophage capsid is packed in a coaxially spooled way with certain writhe bias, then one would observe 
a large proportion of knots and none of these knots would be achiral. In extreme cases one would expect the Figure 8 knot to be missing.

Theorem 3.3 can be strengthened to the following theorem, which relates the writhe of $D_{n}$ to the complexity of $P_{n}^{s}$.

THeorem 3.5. Let $w\left(D_{n}\right)$ be the writhe of $D_{n}$ such that $\left|w\left(D_{n}\right)\right|-\sigma\left(P_{n}^{s}\right)=q \geq 0$, then $P_{n}^{s}$ is a non-trivial knot with minimum crossing number at least $(q+2) / 2$.

Proof. Let us consider the case $w\left(D_{n}\right) \geq \sigma\left(P_{n}^{s}\right)+q$. In this case, $P_{n}^{s}$ must be a non-trivial knot by Corollary 3.4. Let us look a minimum diagram $D$ (which of course is likely to be different from $\left.D_{n}\right)$. It is obvious that $\mathrm{w}(D) \leq \operatorname{Cr}(D)$. Furthermore, we must have $s(D) \leq \operatorname{Cr}(D)$ where $s(D)$ is the number of Seifert circles of $D$. Otherwise, $D$ would be a simple diagram with only twists that can be untwisted using Reidemeister I move repeatedly. Applying Theorem 3 to $D_{n}$ and $D$ separately, we have $w\left(D_{n}\right)-\sigma\left(P_{n}^{s}\right)+1 \leq e \leq E \leq w(D)+s(D)-1$. It follows that $q+1 \leq 2 \operatorname{Cr}(D)-1$. That is, $\operatorname{Cr}(D) \geq(q+2) / 2$ where $\operatorname{Cr}(D)$ is the minimum crossing number of $P_{n}^{s}$ since $D$ is a minimal projection of $P_{n}^{s}$ by our choice. The case of $w\left(D_{n}\right) \leq-(n+q)$ can be similarly handled and is left to the reader as an exercise.

Remark. It is important for us to point out here, that the writhe we talked about in the above discussion refers to the writhe of the diagram of the random polygon (which is the projection of the polygon along the $z$-axis), not the average writhe of that polygon. Theorem 3.3 does not apply, if we use the average writhe instead, because if we project the polygon into a plane that is not parallel to the $x y$-plane, then the number of Seifert circles in that diagram may no longer be the same as the rounding number of the polygon. Therefore, in our simulation study, we would only study the mean of the writhe of the diagram of the polygons into the $x y$-plane, not the mean average writhe. But our result for the crossing number is for the mean ACN.

\section{Numerical results}

As mentioned in the methods section our model has three parameters $R, r$ and $h$, where $R$ and $r$ are the radii of the confining sphere and cylinder, respectively, and $h$ is the parameter that controls the degree of randomness of the polygon at each step and therefore the rigidity of the chain. In order to illustrate the results of the simulations, two values of $h$ are used (i.e. $h=0.1$ and 0.3). The values of $R$ and $r$ are also fixed and results for $R=10$ and $r=1$ are shown. For simplicity the starting point for all polygons is set to be $(1.5,0,0)$. These studies are only our initial efforts to try to understand the model and how the parameters affect our results. Routine studies with systematic testings on these parameters will be performed in the future.

\subsection{The mean radius of gyration of the spooling random polygons: The interplay between $\mathrm{h}$ and $\mathrm{R}$}

In order to test the effects of the parameter $h$ (as defined in Section 2.3) on the dimensions of the knot, we calculated the mean radius of gyration of spooling random polygons. Figure 7 below shows our results. Not surprisingly, polygons generated with $h=0.1$, have conformations tighter than those generated with $h=0.3$. More importantly even for $n=200$, the mean radius

of gyration for the $h=0.1$ case is still far away from $R=10$ (below 3), indicating that most of these polygons are still tightly wrapped around the central cylinder and that the confining sphere did not really play an important role here. However, in the case of $h=0.3$ the mean radius 


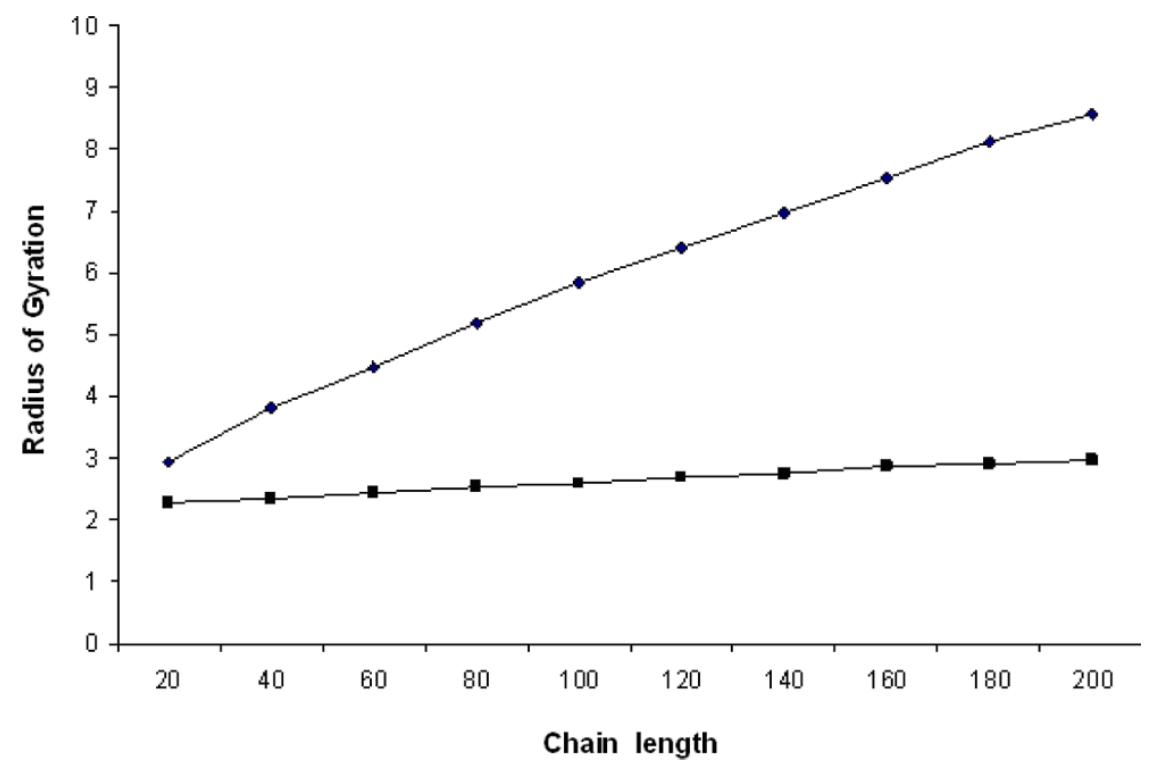

Figure 7. The mean radius of gyration of the spooling random polygons: The bottom curve is for $h=0.1$ and the top one is for $h=0.3$.

of gyration is more than 8.5 when $n=200$, indicating that the polygons could have gone beyond $R=10$ if not confined by the sphere.

\subsection{The knotting probability of the spooling random polygons}

As expected from the tighter structures found for $h=0.1$, the knotting probability increased faster in the case $h=0.1$, than in the case $h=0.3$. Remarkably and in high contrast with other random polygon models the knotting probability rapidly saturates (as it is observed experimentally). However, in contrast with the results published in Refs. [7,44] larger polygons show higher knotting probability than shorter ones. When $w\left(D_{n}\right)$ is large, but not over $\sigma\left(P_{n}^{s}\right)$, how does it affect the knotting probability of $P_{n}^{s}$ ? We suspect that the knotting probability would be much higher for a spooling random polygon $P_{n}^{s}$ with a fixed large writhe $w\left(D_{n}\right)$. A detailed numerical study on this is planned for the future.

\subsection{The mean ACN of $\mathrm{P}_{\mathrm{n}}^{\mathrm{s}}$}

The mean ACN helps to estimate the complexity of the knots in the average sense [41]. Knots extracted from bacteriophage $\mathrm{P} 4$ have been estimated to have an average of 30 crossings [3]. Here, we computed the mean of the ACN before Reidemeister moves were removed, therefore, a much higher number is expected. Figure 9 shows the result of our numerical study on the mean crossing number of $P_{n}^{s}$ (as a function of $n$ ). Polynomials of degree two are fitted to the data as it is suggested in Theorem 3. Interestingly, the mean ACN is heavily dependent on the parameter $h$, suggesting that more complex knots are found for smaller $h$, when the rest of the parameters are held fixed. This is consistent with our numerical result on knotting probability shown in Figure 8.

\subsection{Knot distribution of the spooling random polygons}

Next, we estimated the distribution of knots for up to six crossings. The result for chains of 50 segments and $h=0.1$ are shown in Table 1. Interestingly and in contrast with completely random polygons the distribution shows an elevated number of five crossing knots with respect 


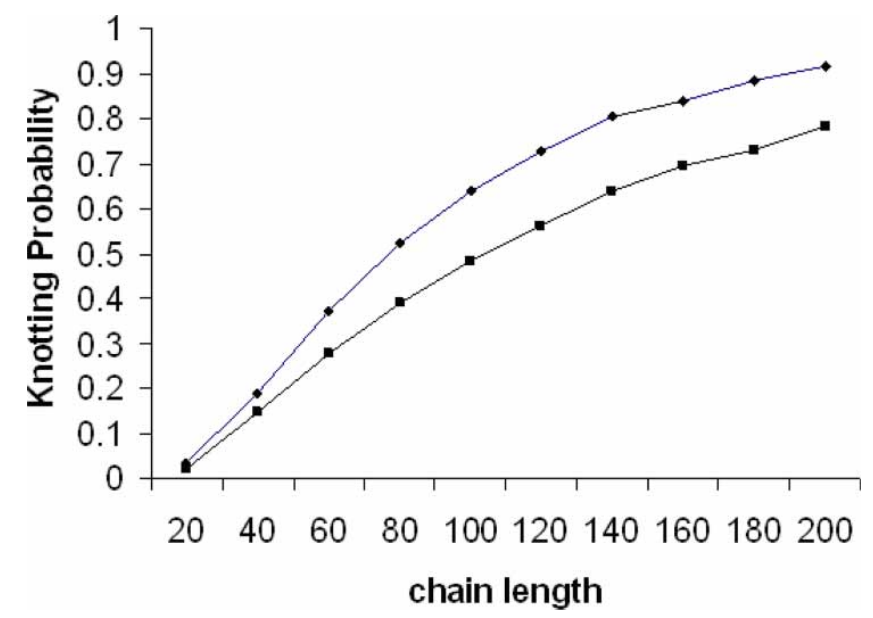

Figure 8. Knot probability of the random spooling model.

to the four crossing knots. It is also important to highlight that (also in contrast with random models) the toroidal knot with five crossings $5_{1}$, is more probable than its twist counterpart. Therefore, the spooling model presents features that are observed experimentally. However, the match between these results and the results observed experimentally is not perfect. For example, figure eight knots do occur (although, with a relatively low percentage). But this should not surprise us, since we did not take out the polygons with small writhe values and Corollary 3 only

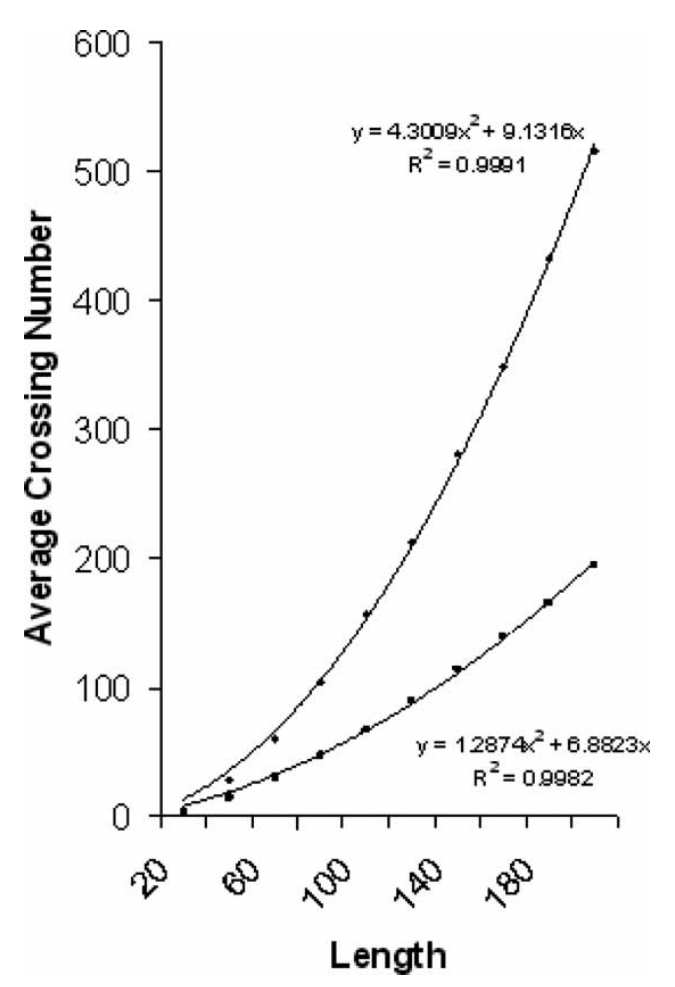

Figure 9. The mean ACN of $P_{s}^{n}$ with $n$ up to 200. 
Table 1. Knot distribution up to six crossings in the case of $h=0.1, n=50$ on a random sample of size 150,000 .

\begin{tabular}{llllllllll}
\hline Knot & $0_{1}$ & $3_{1}$ & $4_{1}$ & $5_{1}$ & $5_{2}$ & $6_{1}$ & $6_{2}$ & $6_{3}$ & $\geq 7$ \\
\hline Probability & 0.64 & 0.157 & 0.012 & 0.017 & 0.016 & 0.001 & 0.002 & 0.001 & 0.07 \\
\hline
\end{tabular}

guarantees us that there will be no achiral knots (such as the figure eight knot) if the absolute writhe of the polygon is large than its rounding number. In our case, the mean absolute writhe is calculated to be about 1.6 which is much smaller than the mean rounding number, which is larger than 5 .

\section{Conclusions}

In this paper, we have introduced a new computational model to investigate DNA knots extracted from bacteriophage P4. This model assumes an initial biased conformation suggested by current models of DNA packing in bacteriophages, and it incorporates a degree of randomness. How much randomness there is in the packing of the genome is a matter of discussion [11,25]. This is an effort to bring together two highly deviated approaches: total randomness or complete organization. As we pointed out in the introduction section, although, both types of idealized models (total randomness or complete organization) have their own merit, they fail to produce numerical results that accurately reflect all aspects of DNA knots extracted from bacteriophage $\mathrm{P} 4$. Therefore, it is important for us to explore new models such as the one proposed here.

Our preliminary study of the random spooling polygons showed that this model can indeed produce numerical results that can partially describe the knotting probability and the knot distribution observed experimentally. Furthermore, because of the special structure of these polygons we are able to carry out some rigorous mathematical analysis. These analytical results give an important support and meaningful interpretation of our numerical results.

Further explorations of the random spooling polygon model will be needed in order for us to understand it better. In particular, more in depth numerical studies will be needed. As we pointed out earlier, we will need to explore the effects of the parameters used in the model in more detail. A big task will be to determine the effect of excessive writhe on the knotting probability.

Additionally, it may also be important for us to impose further conditions on the model due to new geometric or topological restrictions observed experimentally. For example, our current model lacks volume exclusion, however, a number of studies have shown that electrostatics of the DNA chain play an important role in the packing reaction and in the organization of the chromosome (e.g. [16]). Also, in this study, we made several assumptions that will need to be investigated in the future. First, we have introduced an internal cylinder as an ad hoc parameter and also as a model for the core proteins. Second, our polygons are obtained by (artificially) adding a new segment to close the open equilateral chains generated by our method, which may produce artifacts. More realistic rejoining of the chain ends should be investigated.

While some existing theoretical results in knot theory can be nicely applied to the random spooling polygon model as we have demonstrated in Section 3, the model remains a challenge for further mathematical analysis. For instance, if we remove the requirement that the random polygons spool around a central cylinder in the confining capsid, then how can we generalize Theorem 3 and its corollaries (in Section 3)? On the other hand, can we use the average writhe of the polygon instead of the writhe of a particular projection? The reason is that in practice, it is hard to determine what projection plane is the one we need. These are the mathematical issues that we will explore in the future. 


\section{Notes}

1. Supported by NIH grant NIH-2S06GM52588-12.

2. Partially supported by NSF grant DMS-0712958.

\section{References}

[1] C. Adams, The Knot Book: An Elementary Introduction to the Mathematical Theory of Knots, American Mathematical Society, Providence, RI, Revised reprint of the 1994 original 2004.

[2] J. Arsuaga, R.K.-Z. Tan, M. Vázquez, D.W. Sumners, and S.C. Harvey, Investigation of viral DNA packaging using molecular mechanics models, Biophys. Chem. 101 (2002), pp. 475-484.

[3] J. Arsuaga, M. Vázquez, S. Trigueros, D.W. Sumners, and J. Roca, Knotting probability of DNA molecules confined in restricted volumes: DNA knotting in phage capsids, Proc. Natl Acad. Sci. USA 99 (2002), pp. 5373-5377.

[4] J. Arsuaga, M. Vázquez, P. McGuirk, S. Trigueros, D.W. Sumners, and J. Roca, DNA knots reveal a chiral organization of DNA in phage capsids, Proc. Natl Acad. Sci. USA 102 (2005), pp. 9165-9169.

[5] J. Arsuaga, T. Blackstone, Y. Diao, K. Hinson, E. Karadayi, and M. Saito, Sampling large random knots in a confined space, J. Phys. A 40 (2007), pp. 11697-11711.

[6] T. Blackstone, P. McGuirck, C. Laing, M. Vazquez, J. Roca, and J. Arsuaga, The role of writhe in DNA condensation, Proceedings of International Workshop on Knot Theory for Scientific Objects, OCAMI StudiesVol. 1, (2007), pp. 239-250, Osaka Municipal Universities Press.

[7] L. Black, W. Newcomb, J. Boring, and J. Brown, Ion etching bacteriophage T4: Support for a spiralfold model of packaged DNA, Proc. Natl Acad. Sci. USA 82 (1985), pp. 7960-7964.

[8] F.P. Booy, W.W. Newcomb, B.L. Trus, J.C. Brown, T.S. Baker, and A.C. Steven, Liquid-crystalline, phage-like packing of encapsidated DNA in herpes simplex virus, Cell 64 (1991), pp. 1007-1015.

[9] M.E. Cerritelli, N. Cheng, A.H. Rosenberg, C.E. McPherson, F.P. Booy, and A.C. Steven, Encapsidated conformation of bacteriophage T7 DNA, Cell 91 (1997), pp. 271-280.

[10] D.K. Chattoraj and R.B. Inman, Location of DNA ends in P2, 186, P4 and lambda bacteriophage heads, J. Mol. Biol. 87 (1974), pp. 11-22.

[11] L.R. Comolli, A.J. Spakowitz, C.E. Siegerist, P.J. Jardine, S. Grimes, D.L. Anderson, C. Bustamante, and K.H. Downing, Three-dimensional architecture of the bacteriophage phi29 packaged genome and elucidation of its packaging process, Virology 371, pp. 267-277.

[12] T. Dokland, B.H. Lindqvist, and S.D. Fuller, Image reconstruction from cryo-electron micrographs reveals the morphopoietic mechanism in the P2-P4 bacteriophage system, EMBO J. 11 (1992), pp. 839-846.

[13] W.C. Earnshaw and S.R. Casjens, DNA packaging by the double-stranded DNA bacteriophages, Cell 21 (1980), pp. 319-331.

[14] A. Evilevitch, L. Lavelle, C.M. Knobler, E. Raspaud, and W.M. Gelbart, Osmotic pressure inhibition of DNA ejection from phage, Proc. Natl Acad. Sci. USA 100 (2003), pp. 9292-9295.

[15] M.H. Freedman and Z. He, Divergence-free fields: Energy and asymptotic crossing number, Ann. Math. 134 (1991), pp. 189-229.

[16] D.N. Fuller, J.P. Rickgauer, P.J. Jardine, S. Grimes, D.L. Anderson, and D.E. Smith, Ionic effects on viral DNA packaging and portal motor function in bacteriophage phi 29, Proc. Natl Acad. Sci. USA 104 (2007), pp. 11245-11250.

[17] N. Hud, Double-stranded DNA organization in bacteriophage heads: An alternative toroid-based model, Biophys. J. 69 (1995), pp. 1355-1362.

[18] P.J. Jardine and D.L. Anderson, DNA packaging in double-stranded DNA phages, in The bacteriophages., in Richard Calendar ed., Oxford University Press, Oxford, 2006, pp. 49-65.

[19] E. Kellenberger, E. Carlemalm, J. Sechaud, A. Ryter, and G. Haller, Considerations on the condensation and the degree of compactness in non-eukaryotic DNA-containing plasmas, in Bacterial Chromatin: Proceedings of the Symposium 'Selected Topics on Chromatin Structure and Function'., in C. Gualerzi \& C. L. Pon eds., Springer, Berlin, 1986, pp. 11-25.

[20] J.C. LaMarque, T.L. Le, and S.C. Harvey, Packaging double-helical DNA into viral capsids, Biopolymers 73 (2004), pp. 348-355.

[21] J. Lepault et al., Organization of double-stranded DNA in bacteriophages: A study by cryo-electron microscopy of vitrified samples, EMBO J. 6 (1987), pp. 1507-1512.

[22] L.F. Liu, J.L. Davis, and R. Calendar, Novel topologically knotted DNA from bacteriophage P4 capsids: Studies with DNA topoisomerases, Nucleic Acids Res. 9 (1981), pp. 3979-3989. 
[23] L.F. Liu, L. Perkocha, R. Calendar, and J.C. Wang, Knotted DNA from bacteriophage capsids, Proc. Natl Acad. Sci. USA 78 (1981), pp. 5498-5502.

[24] C. Livingston, Knot theory, Carus Math. Monogr. (1993), p. 24, AMS.

[25] C.R. Locker, S.D. Fuller, and S.C. Harvey, DNA organization and thermodynamics during viral packing, Biophys J. 93 (2007), pp. 2861-2869.

[26] C. Micheletti et al., Knotting of random ring polymers in confined spaces, J. Chem. Phys. 124 (2006), p. 064903.

[27] K. Millett, Knotting of regular polygons in 3-space, in Random Knotting and Linking (Vancouver, BC, 1993), World Scientific Publishing, Singapore, 1994, pp. 31-46.

[28] H.R. Morton, Seifert circles and knot polynomials, Math. Proc. Camb. Philos. Soc. 99 (1986), pp. 107-109.

[29] T. Odijk, Hexagonally packed DNA within bacteriophage T7 stabilized by curvature stress, Biophys. J. 75, pp. 1223-1227.

[30] A.S. Petrov, M.B. Boz, and S.C. Harvey, The conformation of double-stranded DNA inside bacteriophages depends on capsid size and shape, J Struct Biol. 160 (2007), pp. 241-248.

[31] G.R. Pruss, J.C. Wang, and R. Calendar, In vitro packaging of satellite phage P4 DNA, Proc. Natl Acad. Sci. USA 71 (1974), pp. 2367-2371.

[32] K. Richards, R. Williams, and R. Calendar, Mode of DNA packing within bacteriophage heads, J. Mol. Biol. 78 (1973), pp. 255-259.

[33] S. Rishov, A. Holzenburg, B.V. Johansen, and B.H. Lindqvist, Bacteriophage P2 and P4 morphogenesis: Structure and function of the connector, Virology 245 (1998), pp. 11-17.

[34] M. Schmutz, D. Durand, A. Debin, Y. Palvadeau, E.R. Eitienne, and A.R. Thierry, DNA packing in stable lipid complexes designed for gene transfer imitates DNA compaction in bacteriophage, Proc. Natl Acad. Sci. USA 96 (1999), pp. 12293-12298.

[35] P. Serwer, Arrangement of double-stranded DNA packaged in bacteriophage capsids: An alternative model, J. Mol. Biol. 190 (1986), pp. 509-512.

[36] D.E. Smith, S.J. Tans, S.B. Smith, S. Grimes, D.L. Anderson, and C. Bustamante, The bacteriophage phi29 portal motor can package DNA against a large internal force, Nature 413 (2001), pp. 748-752.

[37] E.L. Starostin, On the perfect hexagonal packing of rods, J. Phys. Condens. Matter 18 (2006), pp. S187-S204.

[38] S. Trigueros and J. Roca, Production of highly knotted DNA by means of cosmid circularization inside phage capsids, BMC Biotechnol. 7(1) (2007), p. 94.

[39] S. Trigueros, J. Arsuaga, M. Vazquez, D.W. Sumners, and J. Roca, Novel display of knotted DNA molecules by two dimensional gel electrophoresis, Nucleic Acids Res. 29 (2001), p. e67.

[40] S. Tzill, J.T. Kindt, W. Gelbart, and A. Ben-Shaul, Forces and pressures in DNA packaging and release from viral capsids, Biophys. J. 84 (2003), pp. 1616-1627.

[41] A.V. Vologodskii, N.J. Crisona, B. Laurie, P. Pieranski, V. Katritch, J. Dubochet, and A. Stasiak, Sedimentation and electrophoretic migration of DNA knots and catenanes, J. Mol. Biol. 278 (1998), pp. $1-3$.

[42] J.C. Wang, K.V. Martin, and R. Calendar, On the sequence similarity of the cohesive ends of coliphage P4, P2, and 186 deoxyribonucleic acid, Biochemistry 12 (1973), pp. 2119-2123.

[43] S. Wang, J.R. Chang, and T. Dokland, Assembly of bacteriophage P2 and P4 procapsids with internal scaffolding protein, Virology 348 (2006), pp. 133-140.

[44] J.S. Wolfson, G.L. McHugh, D.C. Hooper, and M.N. Swartz, Knotting of DNA molecules isolated from deletion mutants of intact bacteriophage P4, Nucleic Acids Res. 13 (1985), pp. 6695-6702. 


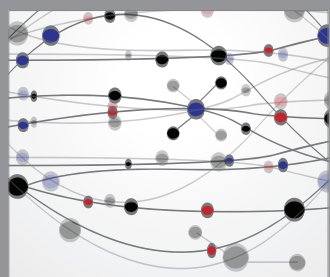

The Scientific World Journal
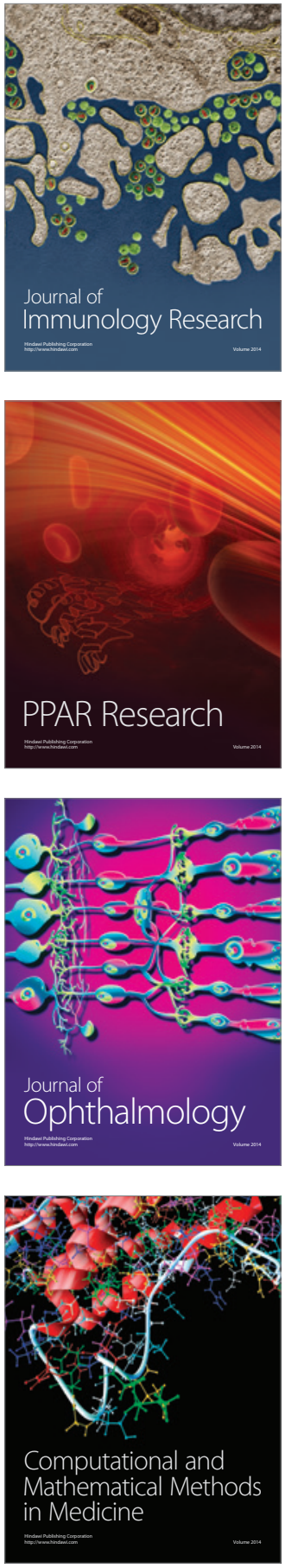

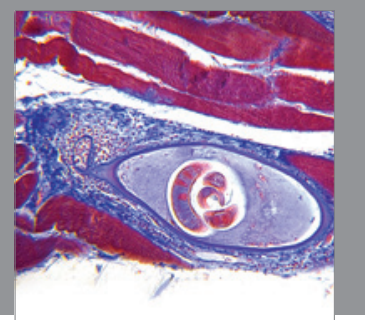

Gastroenterology

Research and Practice
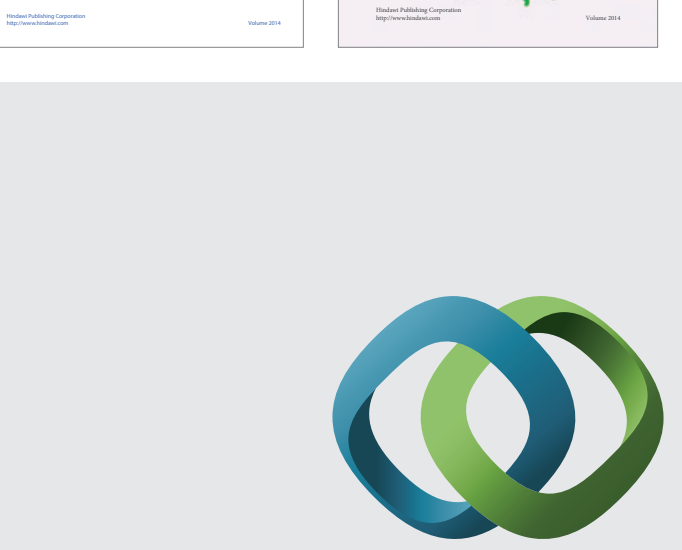

\section{Hindawi}

Submit your manuscripts at

http://www.hindawi.com
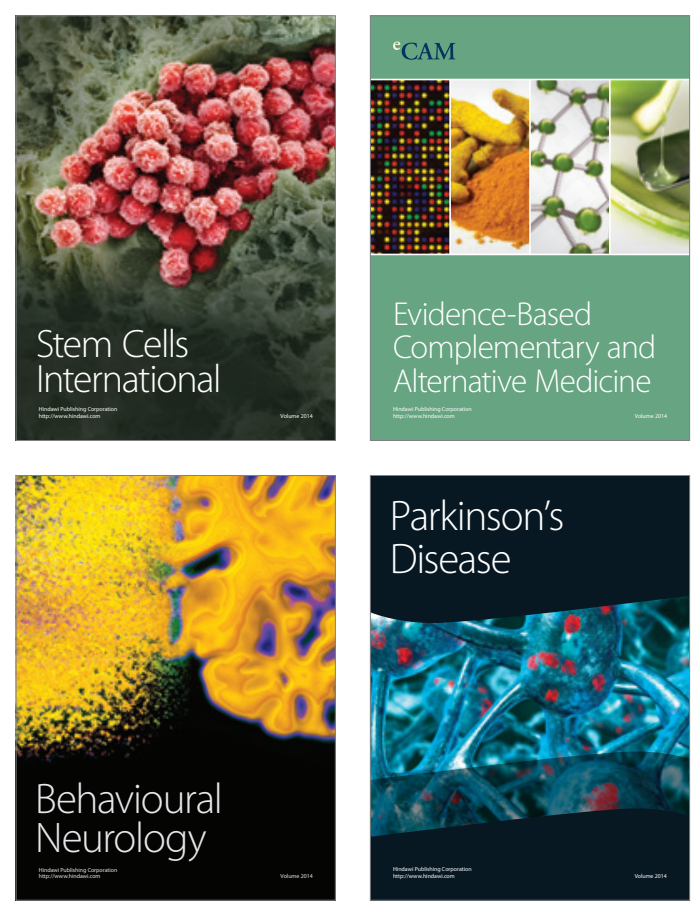

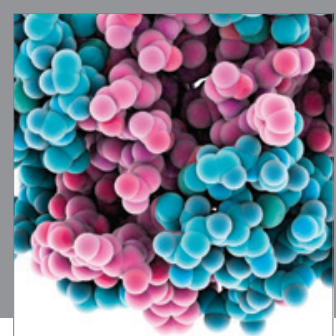

Journal of
Diabetes Research

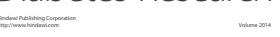

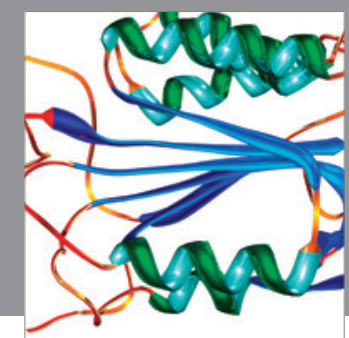

Disease Markers
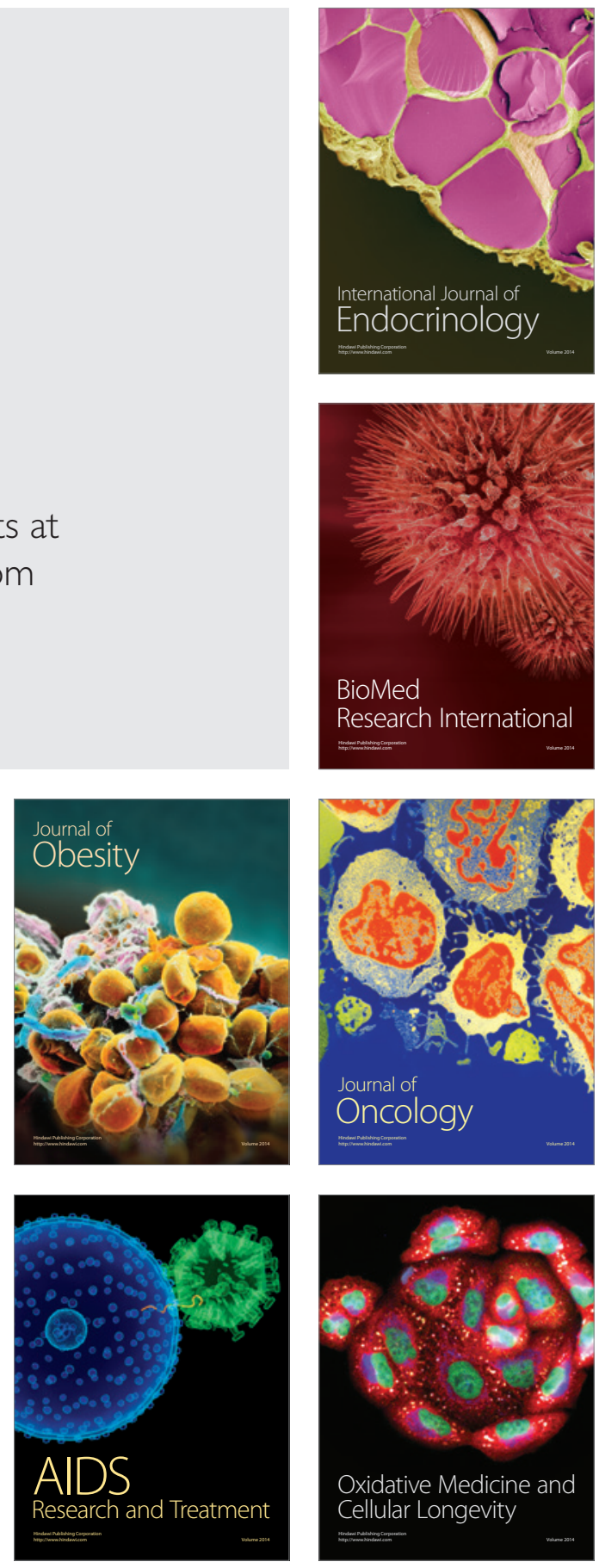